Cinémas

Revue d'études cinématographiques

Journal of Film Studies

\title{
Du cinéma oral au spectateur muet
}

\section{Germain Lacasse}

Volume 9, numéro 1, automne 1998

Les Dispositifs de médiation au cinéma

URI : https://id.erudit.org/iderudit/024772ar

DOI : https://doi.org/10.7202/024772ar

Aller au sommaire du numéro

Éditeur(s)

Cinémas

ISSN

1181-6945 (imprimé)

1705-6500 (numérique)

Découvrir la revue

Citer cet article

Lacasse, G. (1998). Du cinéma oral au spectateur muet. Cinémas, 9(1), 43-62. https://doi.org/10.7202/024772ar

\section{Résumé de l'article}

À ses debuts, le cinéma était oral plutôt que muet, c'est-à-dire qu'il fut rapidement pris en charge par des pratiques tenant encore largement de l'oralité : conférence illustrée, vaudeville, foire. Son dispositif de médiation tenait donc partiellement de pratiques traditionnelles hétérogènes moins disciplinées que ne le sera le cinéma muet, dont c'est le spectateur qui fut graduellement contraint au silence pendant que le film acquérait la faculté d'énonciation. Les vues animées « bonimentées " persistèrent cependant comme tactique résistante dans les communautés produisant peu de films. 


\title{
Du cinéma oral \\ au spectateur muet ${ }^{1}$
}

\section{Germain Lacasse}

\begin{abstract}
RÉSUMÉ
À ses débuts, le cinéma était oral plutôt que muet, c'està-dire qu'il fut rapidement pris en charge par des pratiques tenant encore largement de l'oralité: conférence illustrée, vaudeville, foire. Son dispositif de médiation tenait donc partiellement de pratiques traditionnelles hétérogènes moins disciplinées que ne le sera le cinéma muet, dont c'est le spectateur qui fut graduellement contraint au silence pendant que le film acquérait la faculté d'énonciation. Les vues animées "bonimentées" persistèrent cependant comme tactique résistante dans les communautés produisant peu de films.
\end{abstract}

\section{ABSTRACT}

In its early period, cinema was oral rather than silent, which rneans that it was rapidly subsumed by practices deriving from orality such as the illustrated lecture, the vaudeville show, and the fair. Its apparatus of mediation thus partly took on heterogeneous traditional practices that were less disciplined than silent cinema; spectators were thus only gradually reduced to silence while the film acquired the capacity of utterance. Nevertheless "improved" animated films persisted as a tactic of resistance in communities which produced few films.

\section{Distraction et institution}

La théorie sociologique et esthétique reconnaît généralement que la notion de distraction fait partie intégrante de l'expérience perceptuelle de la modernité (Benjamin, 1969). Celle-ci est 
caractérisée par le choc de la nouveauté, la multiplication et l'intensification de sensations étranges et hétérogènes, la stimulation de la perception et de la cognition par la rencontre d'objets hétéroclites et de récits discontinus. Le cinéma des premiers temps représentait l'essence même de cette nouvelle expérience sensorielle: chaque point du monde connu pouvait être porté à la vue du sujet, selon un dispositif de médiation reproduisant le réel jusque dans son mouvement. Dans l'histoire de la perception et de la cognition, il s'agit d'une révolution absolue. Cependant, cette expérience révolutionnaire a été plus ou moins atténuée au lieu d'être poursuivie et intensifiée. L'institutionnalisation du cinéma s'est faite au détriment de l'expérimentation libre, mais à l'avantage du discours clos; l'institutionnalisation a valorisé et propagé une pratique contemplative du cinéma au détriment d'une pratique divertissante qui survécut néanmoins un certain temps dans les marges. Le cinéma avec bonimenteur appartenait à cette dernière catégorie, et son histoire montre l'émergence d'un nouveau dispositif institutionnalisé.

L'institution ${ }^{2}$ a soutenu l'imposition d'un sujet de la Raison dont l'essence semble contraire à l'expérience de la modernité définie par la distraction, la discontinuité, le choc. Le cinéma primitif proposait un rapport différent avec le réel, en quelque sorte une relation de découverte sans conquête, une confrontation avec l'image mouvante qui modifiait profondément le rapport au temps et à l'espace, comme l'a souligné Marshall McLuhan. Cette relation nouvelle et relativement libre sera donc rapidement balisée par l'intervention des forces se disputant le contrôle et la finalité du cinéma, ces interventions aboutiront à l'institutionnalisation relativement rapide du nouveau dispositif. Les rapports du bonimenteur de film avec cette institution sont complexes et paradoxaux. Dans la plupart des pays, le boniment a eu une fonction préinstitutionnelle: il a préparé l'institution, il a servi d'arbitre ou de surveillant imposant des contrôles encore flous et non encore écrits. Il agit, pour ainsi "dire", à titre d'institution orale avant l'institution écrite.

Mais en même temps qu'il préparait l'institution écrite, le bonimenteur la contrait tout autant. D'une part, il entraînait le cinéma vers une forme de représentation différente, plus tradi- 
tionnelle peut-être, mais où la nouvelle invention suscitait une expérience particulière, plus contextualisée et plus collective. $\mathrm{Si}$ le boniment a survécu plus longtemps dans des pays davantage «attachés» à la tradition, c'est en partie parce que l'expérience nouvelle que constitue la subjectivité contemplative du cinéma institutionnalisé apparaissait déstabilisante ou aliénante pour des collectivités voulant préserver leurs cultures. Le boniment permettait de contrôler et d'adapter sur place, par une institution locale et orale, les textes produits ailleurs. D'autre part, le boniment eut aussi un effet contre-institutionnel - orienté contre l'institution orale même - puisqu'il perpétua souvent et longtemps la possibilité de l'expérience distractive et hétérogène, laquelle est en soi dirigée contre les règles que l'institution impose à l'expérience.

Il semble donc que l'institutionnalisation du cinéma ait soutenu l'émergence d'un cinéma linéaire et littéraire, tandis que le boniment reposait largement sur une expérience orale de l'image animée. D'autres expériences et d'autres pratiques modifieront plus tard l'institution du cinéma, mais jamais plus les films ne seront ainsi présentés par un commentateur qui en faisait son propre texte. Le cinéma d'auteur prendra d'autres formes; le bonimenteur, lui, était un auteur agréé par le public plutôt qu'imposé par l'institution. Nous verrons ici comment ce dispositif particulier constitua une tactique de résistance à l'institution du cinéma.

Après la formation d'une institution cinématographique, c'est surtout dans le mouvement de résistance contre elle que les historiens retrouvent des traces du bonimenteur; même sa fonction préinstitutionnelle est rapidement interrompue lorsqu'il est supplanté par l'objet beaucoup moins aléatoire qu'est le film. Mais qu'est-ce qui constituait une résistance à l'institution? Pourquoi parler de résistance à l'institution? Pour une raison fondamentale: parce que les pratiques où le boniment fut maintenu étaient de nature nettement différente de celles qui furent légitimées. Ces pratiques marginalisées proposaient une autre perception du cinéma: une expérience de type théâtral où l'interprétation est partiellement confiée à des acteurs présents, où le public peut exprimer des réponses qui sont 
entendues; c'est également une situation où il y a interlocution, où la performance est souvent liée à des préoccupations locales et traduite selon une pragmatique particulière variant selon la composition de l'auditoire. On peut synthétiser les caractéristiques de cette expérience par l'expression "cinéma oral" ou "cinéma de l'oralité». La persistance de ces pratiques dans certaines sociétés ou communautés particulières montre que ces dernières appréciaient cette expérience autant que l'autre, voire même la préféraient; il paraît donc justifié de parler de résistance à l'institution. Enfin, dans plusieurs pays et plusieurs formations sociales, ce dispositif marginal revêtait un caractère anticolonial parce qu'il introduisait dans la représentation cinématographique des éléments de la culture locale totalement absents des films eux-mêmes.

La résistance était fondée sur quelques tactiques spécifiques permettant de structurer et d'organiser le détournement de la pratique institutionnelle. Nous en exposons et analysons quatre qui semblent importantes dans la stratégie générale de résistance : l'adaptation de l'explication, la fragmentation du spectacle, la distraction du spectateur et la modification du produit. Toutes aboutissent au même résultat: la conception sur place d'un spectacle empruntant des éléments extérieurs.

\section{L'explication adaptée}

L'explication adaptée, que nous appelons performance pour en distinguer la particularité, fut le principal moyen de résistance fourni par les bonimenteurs contre l'institutionnalisation du cinéma. Si l'intégration du narrateur et la deixis visuelle sont identifiées comme les principales figures du film institutionnel, le boniment, lui, est la figure centrale de résistance, dans la mesure où il est directement opposé à ces procédés. L'évolution du langage cinématographique repose en effet sur la généralisation de figures qui constituent un espace narratif centripète où le narrateur et le sujet sont attirés et intégrés. Tandis que le spectacle de lanterne magique et le cinéma primitif laissaient leurs spectateurs explorer un espace centrifuge sous la gouverne des indications verbales d'un orateur, au contraire l'échelle des plans, les mouvements de caméra, le montage et l'interprétation 
"privée" des comédiens constituèrent un système d'indications visuelles entraînant le spectateur à l'intérieur de l'espace du film. L'espace du spectacle bonimenté comportait plusieurs pôles d'attraction: l'écran, l'orateur, le public, la salle. L'espace du film institutionnel supplantera ces pôles externes en les reconstituant dans la perspective subjective de l'univers diégétique.

La temporalité narrative subit la même série d'opérations. Structurée par les indications verbales du conférencier dans les formes primitives du spectacle d'images, elle sera rapidement prise en charge par le montage, qui fit subir une transformation révolutionnaire à la représentation et la perception du temps. Le temps dit par le boniment devint en quelques années un temps montré par les séquences d'images. Le sujet de la narration orale fut supplanté par un sujet de la vision, le temps dit par un temps vu. Le boniment devint une pragmatique narrative nettement différente de cette nouvelle deixis dont la configuration était assez révolutionnaire, une configuration dont la mutité amplifia peut-être l'étrangeté de l'expérience, privant le sujetspectateur de repères essentiels dans la perception spatio-temporelle, le projetant d'un univers audiovisuel dans un environnement principalement visuel. Le boniment devint, avec la musique et les effets sonores, la principale forme de résistance ou d'adaptation au bouleversement provoqué par la nouveauté de ce dispositif. Mais la hiérarchie de la résistance est moins importante que sa pratique quotidienne et c'est celle-ci qui sera examinée ici. La littérature sur le bonimenteur abonde désormais en exemples de ce phénomène, évidemment plus nombreux dans les pays où le boniment subsiste après l'avènement de l'institution.

En Hollande, la presse assimila le boniment performatif à une pratique populaire empêchant le film d'être un "Art». Plusieurs grands théâtres imposèrent des conférenciers au style plus descriptif, évitant les effets dramatiques créés par leurs collègues qui préféraient manifester leur présence. Les syndicats de bonimenteurs discutèrent cette question, certains critiquant la pratique de leurs collègues plus extravertis. Dans les journaux eut cours un débat très semblable à la polémique menée par le mouvement du Pur Cinéma au Japon: le cinéma ne pouvait être un art 
s'il devait reposer sur la narration externe d'un acteur. Le débat culmina lors d'une exposition internationale de cinéma en 1920; le film de Maurice Tourneur, L'Oiseau bleu, fut présenté avec le commentaire d'un conférencier. Les journaux qualifièrent cette présentation de "performance honteuse " et ceci acheva de chasser le bonimenteur de la plupart des grandes salles (Beusekom, p. 138).

Mais dans les cinémas de quartier et ceux des petites villes, le boniment continua d'être apprécié jusqu’à l'arrivée du cinéma parlant. Le public de ces salles, dont il est établi qu'il préférait le boniment populaire à la conférence cultivée, appréciait donc la prestation de gens comme Nabarro, qui n'hésitaient pas à insérer leurs commentaires ou même à établir des rapports entre la vie de leurs spectateurs et l'histoire montrée à l'écran. Au lieu d'un art racontant une histoire, ce public préférait peut-être une pluralité d'histoires à laquelle le boniment convenait mieux. Cette hypothèse correspondrait aux observations de Jean-François Lyotard sur le différend dans le récit populaire:

Il y a une affinité entre le peuple et le récit. Le mode d'être populaire du "langage» est la petite histoire déritualisée. Petite parce quelle est fidèle aux régimes de phrases et aux différends, que les récits populaires ne cherchent pas à dissiper, mais seulement à neutraliser (p. 228).

Le débat sur la conférence et le boniment, de même que sur la qualité artistique du film sans boniment, semble avoir été mené dans plusieurs pays. Il indique que cette question était importante dans le choix, la hiérarchisation et la légitimation des pratiques du cinéma ; cette discussion montre aussi que malgré la domination officielle de la pratique consensuelle du cinéma, les vues animées persistèrent longtemps comme lieu d'expression de la pluralité des voix. Au Québec, ce débat se présenta peut-être plus tôt qu'en Hollande ou au Japon puisque dès 1908, on remit en cause la compétence du bonimenteur. En 1910, certains le croyaient déjà archaïque. Pourtant, de nombreux comédiens continuent de commenter des films en 1925, dans des spectacles dont nous ne connaissons pas toujours la teneur mais où le mélodrame et la parodie semblent importants 
et paraissent l'un et l'autre aussi éloignés du propos des films "artistiques" de cette période 3 .

Alexandre Silvio peut faire une "[...] conférence sur ce photodrame sensationnel ${ }^{4}$ de Pathé qu'est L'Enfant de la vieille fille, mais il fait aussi des "comédies-conférences " 5 sur d'autres films dont il continue d'adapter les titres. Silvio est un des seuls bonimenteurs dont la critique mentionne le nom et commente quelque peu le travail. Il y avait probablement là une part de complaisance envers le travail d'un animateur important, mais il est visible malgré cela que sa production tenait plus du boniment populaire que de la conférence savante, surtout à partir du moment où il insère sa prestation dans un spectacle dont la forme burlesque est évidente et avouée. Les journaux montréalais des années vingt ne semblent avoir mentionné, outre lui, que Léopold Gosselin et Omer St-Georges dont le travail n'est cependant pas commenté; mais d'autres, dont Pierre Desrosiers et Alex St-Charles, exerçaient aussi leur métier qu'on évoquera 30 ans plus tard en parlant de "savoureux commentaires de vues animées" (Béraud, p. 129).

\section{La fragmentation du spectacle}

Le bonimenteur était une instance de type théâtral, mais sa prestation n'était pas le seul moyen de résistance à une pratique plus linéaire et plus passive du cinéma. Très souvent, sa performance faisait partie d'un spectacle composite où il pouvait être le présentateur de diverses attractions, ou demeurer simplement le bonimenteur de films. Une large fraction des bonimenteurs étaient des gens de spectacle aux multiples ressources; ils étaient non seulement comédiens, mais souvent monologuistes, chanteurs et musiciens. Leur capacité convenait fort bien au type de spectacle fragmenté et hétérogène qui apparut avec la multiplication des salles de cinéma. Ne fut-ce que pour changer les bobines et laisser reposer le projectionniste qui opérait des machines entièrement manuelles, les projections exigeaient des interruptions; la mutité des films semblait insupportable sans musique, et leur histoire souvent confuse sans explication. Toutes ces raisons justifiaient la présence d'un commentateur, mais aussi celle de musiciens, de chanteurs, d'amuseurs de toutes sortes. 
L'arrivée de films plus longs permit de mettre à pied une bonne partie de ce personnel. Plusieurs d'entre eux ont néanmoins gardé leur emploi pendant longtemps, probablement parce que cette forme hétérogène de spectacle correspondait plus au goût d'un certain public, ou à un certain goût du public, au point que plusieurs producteurs investiront dans ce genre pendant deux décennies, parfois plus longtemps: au Québec, 10 ans après l'arrivée du cinéma sonore, des salles présentaient encore un spectacle de variétés où des chanteurs comiques et des comédiens de burlesque succédaient à un film (Hébert, 1981 ; Pétrie, 1977). Évidemment ils avaient commencé bien plus tôt, on peut retracer cette pratique jusque dans les spectacles de lanterne magique où le conférencier et le bonimenteur étaient soutenus par un pianiste ou un orgue de barbarie. Dès l'ouverture en 1906 du Ouimetoscope, premier cinéma au Québec, les films sont entrecoupés de chansons illustrées, d'airs d'opérette ou de chansons comiques. Les autres salles suivent vite cet exemple emprunté aux États-Unis, d'où viennent une bonne partie des films et des artistes.

Puisqu'il est question ici de résistance à l'institution, il faut considérer l'évolution de ces formes au moment où l'institution apparaît et se développe. Or, au Québec, c'est au moment de la consolidation de l'institution, pendant la guerre de 1914-1918, qu'apparaît et se consolide le burlesque québécois dont nous savons qu'il était constitué en partie par des films bonimentés. Pendant que se développent d'un côté des salles plus spécifiquement adaptées au cinéma, dans lesquelles sont présentés des films plus longs accompagnés par un orchestre et comportant des intertitres en Anglais uniquement (fait d'importance majeure), dans des salles plus petites, plus anciennes, et fréquentées surtout par la communauté francophone, est développé par ailleurs un type de spectacle qui poursuit ou même amplifie encore l'hétérogénéité des premières représentations. Loin de se résorber, cette forme divergente paraît même s'être consolidée après quelques années. On peut en juger en examinant un programme du théâtre Canadien-Français en 1919:

Programme des plus attrayants, quelques rouleaux de vues drôles, Montréal la nuit, scène d'actualité par la 
troupe Castel, 10 personnes en scène, chansons et chœurs. [...] En plus, programme de chansons et vues spéciales expliquées par le célèbre conférencier Alexandre Silvio .

La formule fonctionne très bien, puisque le CanadienFrançais se vante d'être "[...] le seul théâtre qui ait pu réussir à rester ouvert été comme hiver ${ }^{7}$ ". La présentation évolue et devient plus éclatée encore. On ajoute des parodies par des acteurs pendant que Silvio explique les vues, des émissions de radio transmettant la prestation d'artistes montréalais dans d'autres villes, des monologues et des chansons, même quelques-uns des rarissimes films de fiction tournés à Montréal pendant les années vingt, en plus d'un défilé constant des plus populaires artistes du vaudeville et du burlesque. En 1923, quand un spectateur achète un billet du théâtre Canadien ou du théâtre National, il voit une revue d'actualités écrite par Castel ou Robi, une opérette chantée par Aurore Alys ou Damase Dubuisson, un film bonimenté par Silvio, Desrosiers ou Gosselin, accompagnés au piano par Pellerin, Foucreau ou Lafrenière, une ligne de danseuses conduites par Effie Mack ou Henriette Demers, et enfin des monologues et chansons comiques interprétés par Leclaire, Pellerin ou Desmarteaux. Ce spectateur a vu quelques films intégrés dans un spectacle théâtral, mais aussi un spectacle théâtral intégré dans des films, puisqu'en plus des commentaires, il a eu droit aux comédies-conférences de Silvio ou aux vues parodiées par Castel et Gauthier simultanément à leur interprétation par Silvio et par: un pianiste, auquel on demandait certainement d'être complice de cette spectaculaire machination. Ce spectateur en avait probablement pour son argent!

Les territoires comme le Québec n'eurent pas le monopole de ce genre de spectacle composite; il y occupa peut-être une place plus importante ou une position plus influente, mais dans les pays producteurs de films, de telles formes furent fréquentes, même si elles purent être moins remarquées. À Chicago, des pièces polonaises étaient présentées avec des films américains et dans les quartiers italiens, la musique de ce pays était l'accompagnement le plus fréquent des films américains, tout comme dans les ghettos noirs, le jazz et les attractions typiques de la culture 
afro-américaine faisaient souvent partie du spectacle (Cohen, p. 16; Hansen, p. 100). L'historien américain Richard Koszarski prétend quaux États-Unis, durant toute la période du cinéma muet, aller au cinéma était une expérience théâtrale: " [...] essentially a theater experience, not a film experience» (p. 99). Un tiers des programmes était consacré à des attractions autres que le film principal (qui était même parfois coupé ou projeté plus rapidement), des numéros de vaudeville, des chansons illustrées, des concerts par l'orchestre du théâtre, en plus des films d'actualités et des courts films comiques. Cette conception du spectacle était apparemment extrêmement populaire; Koszarski mentionne des sondages faits tout au long des années vingt révélant que 10 pour 100 seulement des spectateurs venaient pour le film, les autres se déplaçant surtout pour le spectacle, "the event».

Peut-être Koszarski exagère-t-il un peu. Ces spectacles étaient nettement moins proches du théâtre que ceux présentés à Montréal, Amsterdam ou Tokyo, on n'y trouvait ni bonimenteur, ni pièce de théâtre, mais le spectacle reposant principalement sur les films était cependant encore fragmenté et discontinu, même si c'était à un degré moindre que dans les pays susmentionnés. L'intégration du spectateur à un univers diégétique monologique n'était pas encore achevée, même si le récit filmique reposait maintenant principalement sur les figures cinématographiques. Le film sonore permettra aux instances de production d'assurer encore davantage leur contrôle sur le spectacle, mais les attractions accompagnant le film ne disparaîtront que lentement.

\section{La distraction du spectateur}

Le concept de distraction fait partie des caractéristiques que des sociologues (Simmel) et des philosophes (Benjamin) ont attribué à la modernité. Il désigne en particulier la sensation de choc et d'excitation intense provoquée par la surprise des nouveautés techniques concentrées dans un espace urbain où la consommation est intensifiée. Le cinéma correspondait exactement à l'essence de cette expérience, puisqu'il était un dispositif déstabilisant la conception courante de l'espace et du temps de son 
spectateur et simulant devant lui des situations et des événements auparavant presque impossibles à expérimenter. Les premiers films étaient en eux-mêmes des attractions, dont l'étrangeté fut probablement atténuée par les commentaires des conférenciers ou bonimenteurs dont la voix rétablissait une certaine familiarité. Le spectacle cinématographique évolue assez rapidement vers une intégration de l'attraction à l'univers diégétique: les tensions, les surprises et les chocs venant de films comme The Lonedale Operator (D. W. Griffith, 1911) sont des exemples probants, au même titre que les attractions qu'Eisenstein définira comme "éléments de pur spectacle". Mais cette intrégration de l'attraction à l'univers diégétique était déjà étrangère à l'expérience de l'attraction dans le contexte oral qu'étaient les vues animées bonimentées.

Une métaphore couramment exploitée dans le premier cinéma et dans les autres médias au début du siècle exprimait bien l'importance de la rupture avec l'expérience antérieure: la caricature, sous une forme ou une autre, du campagnard visitant la ville et se couvrant de ridicule parce quil ne connaît presque rien des choses qu'il voit. Cette métaphore a été exploitée très tôt dans le cinéma américain, entre autres en 1902 dans un film d'Edwin Porter: Uncle Josh at the Moving Pictures. Elle fut aussi illustrée dans le spectacle québécois sous diverses formes, dont un monologue de Paul Coutlée, L'Oncle Bernabé aux vues animées:

Après, su la toile, j'ai vu un individu qui massacrait la plus belle p'tite criature que j'ai jamais vue d'ma vie. J'me lève pis j’y crie: "Cout' donc, toé, vas-tu la lâcher c't'fille-là?». Mais c'est pareil comme si j'avais pas parlé. Alors j'grimpe su l'stage, pis à grands coups d'mon parapluie... j'crève la toile. Le lend'main Polion est venu me sortir de la station. $Y$ m'avaient fourré au poste. Allez donc rendre service au monde! Ah, $j$ 'suis pas prêt de r'tourner à Marial, ni aux vues envlimées non plus. À r'voir! (p. 83).

L'incompétence ou l'ignorance du fermier visitant le scope est ridiculisée par le citadin habitué à cette expérience; des textes très semblables traiteront du train, de l'automobile, de diverses 
inventions courantes dans la vie urbaine, mais qui ont bouleversé rapidement l'expérience humaine. Telle était l'expérience de la modernité: "La modernité, de quelque époque qu'elle date, ne va jamais sans l'ébranlement de la croyance et sans la découverte du peu "de réalité" de la réalité, associée à l'invention d'autres réalités" (Lyotard, p. 363).

Cette expérience révolutionnaire du réel fut celle du premier cinéma, peut-être surtout du film vraiment muet et incolore, quand aucune voix ni aucune musique ne l'accompagnaient; Maxime Gorki a exprimé cette sensation étrange, dans un texte désormais célèbre intitulé "Au royaume des ombres" ". Le cinéma des débuts reposait largement sur cette expérience, qu'André Gaudreault et Tom Gunning ont très justement baptisée «cinéma des attractions» (p. 59). Cette expérience et ses éléments ont été assez rapidement dilués par la narrativisation du cinéma, son intégration dans des spectacles narratifs linéaires; l'institution a légitimé ce genre de spectacle, mais n'a pas fait disparaître les autres genres qui ont perduré dans les marges". Ces spectacles fondés sur l'attraction et sur la distraction furent d'autres formes de résistance à l'institution, auxquelles le bonimenteur apporta de nouveau sa contribution.

Il faut probablement classer dans cette catégorie de tactique de résistance la propension à traiter et à annoncer comme nouveauté divers sujets cinématographiques ou divers éléments de spectacle comme les essais de cinéma sonore. La notion de nouveauté, de novelty, repose sur la distraction et la surprise que le film intégrera à la diégèse, mais qui est ici extradiégétique. Les films d'actualités, dont la projection dans les salles de cinéma continuera pendant très longtemps, faisaient partie de ce dispositif, car ils étaient très différents du cinéma documentaire, qui deviendra un genre spécifique fondé sur des conventions précises et légitimé par un discours particulier. Les films d'actualités continuaient d'exploiter la nouveauté sous forme d'insolite, de surprise, d'étrange, poursuivant l'expérience amorcée dans les toutes premières projections de cinématographe. Mais cette intrégration de l'attraction dans l'univers filmographique était déjà étrangère à l'expérience de l'attraction dans le contexte oral qu'étaient les vues animées bonimentées. 
Au Japon, la très longue persistance du boniment prolonge l'existence du film comme attraction externe encadrée dans le spectacle théâtral; de toutes les manières possibles, la société japonaise résiste à l'autonomie du spectacle cinématographique, à la constitution d'un espace énonciatif abstrait reposant sur l'expérience de la modernité. Pendant presque quatre décennies, le spectacle filmique nippon fonctionne comme le cinéma des attractions occidental, et même les films produits dans ce pays demeurent assez foncièrement différents de ceux de la production étrangère, comme l'ont montré plusieurs historiens (Komatsu, Burch, Anderson). Toutes les formes du spectacle de benshi sont structurées autour d'une narration verbale dont le film est l'illustration et l'attraction. Même le kakeai (ou kowairo), spectacle où des comédiens cachés prêtent leur voix aux personnages, revêt une forme principalement orale et thêâtrale, comme on peut le voir dans ces répliques écrites par un benshi et rapportées par Komatsu et Loden:

Scène '́́. Le Samourai itinérant et l'exilé soupçonneux. Un exilé (âgé de 30 ans) gît malade sur la route. Jiraiya (25 ans) s'approche et lui parle.

Jiraiya: Je dis, que t'arrive-t-il, exilé? Tu sembles souffrir de quelque maladie. Tu dois être très affecté. (intonation chantante) Voici de l'argent pour acheter de quoi manger et te soigner (il lui offre de l'argent).

L'exilé: (il repousse péniblement l'argent) J'apprécie beaucoup votre compassion. Comme vous le voyez cependant, je suis malade et ne peux me lever. Même si je prenais cet argent, je ne pourrais aller acheter quoi que ce soit, alors je ne peux accepter. Si vous voulez vraiment m'aider, donnez-moi plutôt un remède ${ }^{10}$.

Ce texte ressemble beaucoup plus à celui d'une pièce qu'à un dialogue de film et l'image y est vraiment traitée comme un ajout: "Comme vous le voyez, je suis malade..." dit le second personnage; son état est probablement visible à l'écran, mais pourtant l'introduction le dit, l'autre personnage le répète et luimême le redit à son tour en ajoutant encore que son état est visible. La répétition exagérée semble être le moyen de maintenir la position énonciative du bonimenteur et de la refuser au film qui n’est dès lors qu'un élément attractif du spectacle théâtral. 
Les Jordaan Films hollandais, films muets bonimentés adaptés d'œuvres du théâtre local (Blom et van Dooren, p. 87) peuventils être considérés comme des attractions? Ils ont comme sujet la vie des quartiers populaires, mais cette vie mise en images a valeur d'attraction et de nouveauté autant que de récit; le spectacle n'est pas tant cette vie connue que l'acte de sa récitation simultanée par le film et par le bonimenteur. La mieux connue des expériences du boniment, outre celle du Japon, est l'expérience hollandaise, qui semble présenter plusieurs points de similitude avec la japonaise. Le boniment n'y demeure pas légitimé aussi longtemps, mais il assure là aussi l'arraisonnement, la mainmise de la tradition orale sur l'attraction cinématographique maintenue comme élément hétérogène du spectacle.

La plus courante manifestation de la distraction dans la résistance au spectacle institutionnel, c'est probablement dans l'attitude du public qu'elle est visible et c'est toujours au boniment qu'elle est liée. Tandis que le spectacle cinématographique évolue vers une forme monologique d'où l'intersubjectivité est pratiquement bannie, le spectacle bonimenté perpétue une forme de rencontre où l'interlocution est tolérée sinon encouragée. Comme l'écrivait le critique et conférencier américain Stephen Bush, la voix du conférencier fait taire celle des spectateurs; mais son texte relatait un souhait autant qu'une réalité: la voix interne se tait rarement et trouvait assez fréquemment à se manifester. Elle en avait moins souvent l'occasion dans les salles où présidaient des conférenciers, mais semble avoir été tolérée davantage dans les espaces plus modestes où se produisait le bonimenteur. L'exemple extrême est ici la prestation des bonimenteurs zaïrois contemporains rapportés par Mpungu Mulenda Saïdi : connus du public et affublés de surnoms familiers, les "Western", "Aznavour», "Papy Joe» et consorts commentent le film, mais dans un dialogue continu avec les gens de la salle (Saïdi, p. 128-129).

Si c'était l'exemple extrême, cela ne signifiait pas qu'il fût rare: il faudrait alors oublier les séances de "parole vivante" (Zivoe Slovo) en Union soviétique, les projections dans les cinémas iraniens et les théâtres de bien d'autres pays semblables, le tumulte des salles foraines et toute la familière cacophonie 
montrée cent fois au cinéma par les cinéastes (Fellini, Tornatore, etc.), mais qui n'est pas une fable même si elle peut sembler fabuleuse.

\section{La modification du produit}

Cette dernière tactique ne fut pas inventée par le bonimenteur de la péricide de résistance, elle fut plutôt empruntée aux premiers exploitants et développée par leurs successeurs. C'est l'historien Charles Musser qui en a expliqué le principe. Selon lui, la postproduction des premiers films se faisait fréquemment dans la cabine de projection, parce que chaque exploitant organisait à sa guise les éléments du spectacle. Les films n'avaient souvent qu'un seul plan, et celui qui projetait pouvait décider de leur agencement comme bon lui semblait. Le boniment devenait encore plus important si l'exploitant n'achetait qu'une partie des courts films composant une série: «Plusieurs de ces scènes étaient mal connues et, de ce fait, leur intelligibilité et leur signification reposaient davantage sur le commentaire d'accompagnement" (Musser, p. 17).

Cette tactique sera sans doute atténuée par la durée de plus en plus longue des films, mais elle sera utilisée longtemps dans tous les «territoires bonimentés». L'exemple le plus frappant est celui de Libansky, un exploitant polonais qui faisait des conférences sur l'astronomie en montrant les films fantaisistes de Georges Méliès (Hendrykowska, p. 166). Il y eut des centaines, des milliers de représentations où ce principe était appliqué de façon plus ou moins radicale. Le Japon peut paraître encore l'exemple le plus singulier, mais cette singularité d'origine ethnocentrique est vite atténuée quand on découvre des exemples semblables dans les pays occidentaux aux traditions différentes, mais qui offraient la même diversité formelle que le spectacle japonais. La projection de film avec benshi était divisée en plusieurs sous-genres où le film n'était jamais utilisé de la même façon:

Tandis que le bonimenteur d'un petit théâtre ou d'un spectacle ambulant devait pouvoir commenter chaque sorte de film, la plupart des autres se spécialisaient dans l'un des trois genres du métier: jidai-geki (récit japo- 


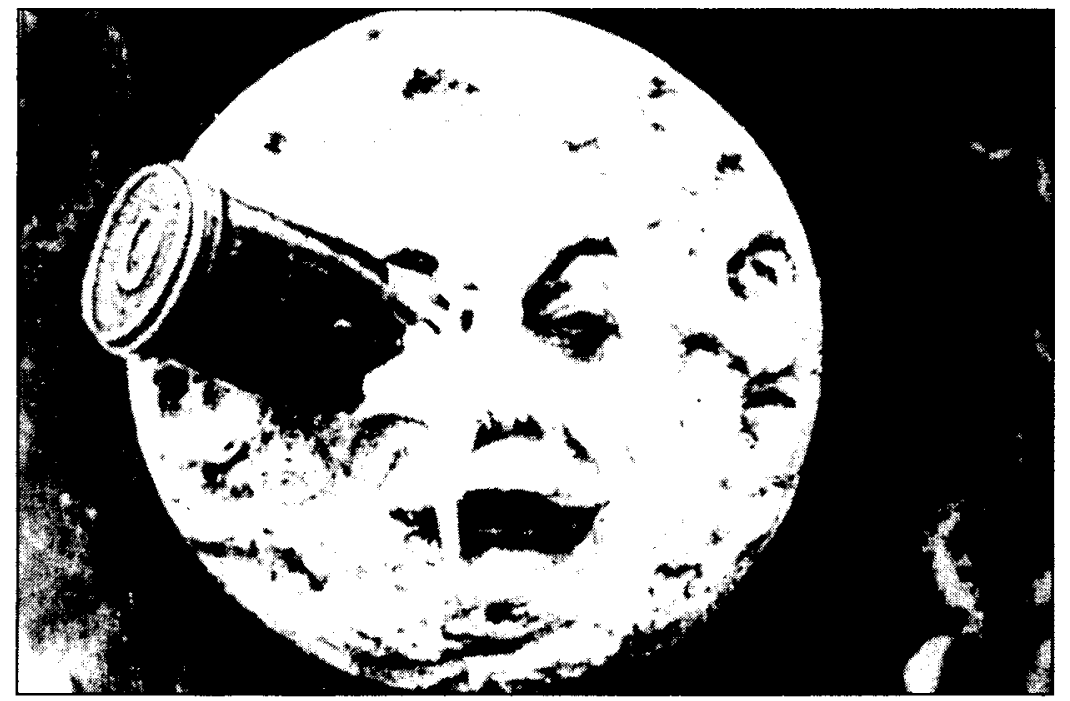

\section{Le Voyage dans la lune de Georges Méliès (1902)}

Collection Cinémathèque québécoise

nais historique), gendai-geki (récit japonais contemporain) et yoga (film étranger). Les bonimenteurs les plus versatiles tenaient cependant à exercer leur talent dans les trois catégories (Anderson, p. 274).

Des bonimenteurs européens semblent avoir développé la même versatilité et s'être permis autant de liberté par rapport au film. Cette hypothèse aurait semblé invraisemblable il y a peu, mais elle est maintenant fondée sur la recherche des historiens et appuyée par les récits de plusieurs auteurs fascinés par le cinéma. Blaise Cendrars relate ainsi la performance d'un bonimenteur appelé M. Nouvel An. Celui-ci semble avoir eu des capacités fabuleuses pour imiter les voix et les bruits. Il y met tant de talent et d'ardeur que sa prestation semble pour l'auditeur aussi fascinante que celle du film, même quand il s'agit d'une comédie jouée par un maître comme Chaplin :

M. Nouvel-An était le directeur de ce cinéma-bistro.

[...] Pour les films de Charlot il improvisait des sketches vertigineux et irrésistibles. Sa voix imitait toutes les contorsions, toutes les acrobaties de Charlot et de 
ses comparses. [...] Le petit chapeau, le large pantalon, la petite canne, la fuite, les chutes, les glissades, l'équilibre instable, la soif, la faim, l'amour, chacune des particularités de Charlot avait sa voix propre, son intonation spéciale, son accent et son timbre".

Non content d'interpréter vocalement et gestuellement Chaplin, M. Nouvel An insère son film dans un spectacle dont il a choisi les autres attractions afin de mettre en valeur son talent d'improvisateur. C'est son spectacle autant que celui de Charlot. Les bonimenteurs n'avaient sans doute pas tous cette verve et cette versatilité, mais la liste de ceux qui prenaient ainsi la vedette pourrait être assez longue; après tout, n'était-ce pas ce qu'on attendait des milliers de gens de scène qui exercèrent ce métier un peu partout dans le monde? On pourrait citer plusieurs exemples québécois pour prolonger la démonstration, mais ils ont déjà été mentionnés: les vues bonimentées par Silvio et parodiées par Castel, les spectacles de burlesque des troupes Pétrie ou Guimond, les revues accompagnant des films et traitant du cinéma... Comme les gens si "étranges" dont ils étaient séparés par un continent et un océan, les Canadiens français intégraient le cinéma à un spectacle théâtral de conception locale dans lequel le film était inséré et qui ne pouvait manquer de déterminer sa lecture. Vu sous cet angle, le boniment et les pratiques similaires ne peuvent être conçus autrement que des dispositifs résistants puisqu'ils font partie de l'arsenal de moyens dont l'institution du théâtre paraît s'être dotée pour lutter contre celle du cinéma.

Cette résistance n'est cependant pas celle d'un commerçant luttant contre un rival, même s'il y entre nécessairement des considérations d'ordre économique. C'est plutôt la manifestation de formes de subjectivité cherchant à échapper à la pragmatique visuelle propagée par le cinéma muet, qui assure la monopolisation d'un spectacle, mais en même temps la popularisation d'une deixis balayant les systèmes antérieurs de représentation. Intégrer le film à un spectacle théâtral, c'était restreindre son pouvoir révolutionnaire en préservant les habitudes et les règles narratives que le cinéma avait transformées en l'espace de quelques années. Comme le pensait Marshall McLuhan, le 
média est doté d'une force bien plus grande que ne le pensent ceux qui attribuent plutôt sa portée à l'usage qui en est fait: «Le cinéma, tout autant que l'alphabet et l'imprimé, est une forme agressive et impérialiste dont l'expansion explosive pénètre les autres cultures [...]» (McLuhan, p. 322). En face de ce grand pouvoir du média, McLuhan s'étonnait et se réjouissait de voir des forces de résistance comme celles que représentaient les bonimenteurs thaillandais qu'il mentionnait et auxquels il attribuait des créations plus originales que l'institution en marge de laquelle ils travaillaient. Face à la médiation nouvelle qu'introduisent les dispositifs apparaissent souvent des pratiques de médiation du dispositif lui-même. Cette dialectique étonne moins si on évoque la fonction de la médiatrice: coupant un espace géométrique en deux, elle fait surgir deux univers distincts.

Université Laval

\section{NOTES}

1 Ce texte a été écrit dans le cadre des travaux du GRAFICS (Groupe de recherche sur l'avènement et la formation des institutions cinématographique et scénique) de l'Université de Montréal, subventionné par le Conseil de recherches en sciences humaines du Canada et le Fonds pour les chercheurs et l'aide à la recherche du Québec.

2 Notre définition de l'institurion est celle proposée par Roger Odin: "Une structure articulant un faisceau de déterminations" ("Pour une sémio-pragmatique du cinéma ". Iris, vol. $1, \mathrm{n}^{\prime \prime} 1,1983$, p. 71). Odin cite aussi la définition de Berrendonner: "Un pouvoir normatif assujettissant mutuellement les individus à certaines pratiques sous peine de sanction" (Éléments de pragmatique linguistique. Paris: Éditions de Minuit, 1981, p. 75).

3 Voir notre article «Du boniment québécois comme pratique résistante» (Iris, n" 22, 1996, p. 53 à 66).

4 Anonyme, "Théâtres. Au Théâtre Canadien", La Patrie, Montréal, 17 mai 1919.

5 Anonyme, "Théâtres. Au Canadien-Français", La Patrie, Montréal, 7 juin 1919 ; Anonyme, "Théâtre Canadien ", La Presse, Montréal, 28 juin 1919.

6 Anonyme, "Théâtres. Au Canadien-français", La Patrie, Montréal, 7 juin 1919.

7 Anonyme, "Théâtres. Au Canadien-français", La Patrie, Montréal, 8 juillet 1919. À cette époque, à cause de la chaleur suffocante dans les salles et des vacances estivales d'une partie de la clientèle, plusieurs théâtres fermaient pour l'été.

8 Le texte de Maxime Gorki est cité dans l'ouvrage de Jay Leyda, Kino. Histoire du cinéma russe et soviétique (Lausanne: L'Âge d'homme, 1976, p. 472-474).

9 Jean-Luc Godard, dans une interview dont les coordonnées sont perdues, disait avec pertinence que «les marges tiennent les pages". 
10 Anonyme, texte d'accompagnement du film Jiraiya, (Kokkatsu, 1921), cité par Komatsu et Loden dans "Maîtriser l'image muette: le rôle des benshis dans le cinéma japonais" (Iris, n"22, 1996, p. 40).

11 Le texte de Blaise Cendrars, Les Confessions de Dan Yack, est cité dans l'ouvrage de Jérôme Prieur, Le Spectateur nocturne. Les écrivains au cinéma. Une anthologie (Paris: Cahiers du cinéma, 1993, p. 105).

\section{OUVRAGES CITÉS}

Anderson, J. L. "Spoken Silents in the Japanese Cinema; or, Talking to Pictures: Essaying the Katsuben, Contexturalizing the Text», dans Arthur Nolletti et D. Desser (direction), Reframing Japanese Cinema: Authorship, Genre, History. Bloomington: Indiana University F'ress, 1992.

Anderson, Joseph L, et D. Richie. The Japanese Film Art \& Industry. Rutland : C.E. Tuttle, 1959.

Benjamin, Walter. "L'œuvre d'art à l'époque de sa reproduction mécanisée », CEuvres I. Mythe et violence. Paris: Denoël, 1969.

Béraud, Jean. 350 ans de théatre au Canada Français. Montréal: Le Cercle du Livre de France, 1958.

Berrendonner, Alain. Éléments de pragmatique linguistique. Paris: Éditions de Minuit, 1981.

Beusekom, Ansje van. "The rise and fall of the lecturer as entertainer in the Netherlands. Exhibition practices in transition related to local circumstances". Iris, n" 22 (1996), p. 131-144.

Blom, Ivo, et Ine van Dooren. "Ladies and gentlemen, hats off, please! Dutch Film Lecturing and the Case of Cor Schuring ". Iris, n" 22 (1996), p. 87-101.

Cohen, Lizbeth. "Encountering Mass Culture at the Grassroots: The Experience of Chicago Workers in the 1920s". American Quarterly, vol. 4l, n"1 (1989), p. 6-33.

Coutlée, Paul. Que nous dis-tu. Montréal : éditeur inconnu, 1922.

Gaudreault, André, et T. Gunning. "Le cinéma des premiers temps: un défi à l'histoire du cinéma?", dans Jacques Aumont et al. (direection), Histoire du cinéma. Nouvelles approches. Paris: Publications de la Sorbonne (1989), p. 49-63.

Gaudreault, André, et D. Simard. "L'extranéité du cinéma des premiers temps : bilan et perspectives de recherche", dans Jean A. Gili et al. (direction), Les Vingt premières années du cinéma français. Paris: Presses de la Sorbonne Nouvelle-AFRHC (1995).

Hansen, Miriam. Babel and Babylon. Spectatorship in Early Silent Film. Cambridge: Harvard University Press, 1991.

Hébert, Chantal, Le Burlesque au Québec. Un divertissement populaire. Montréal: Hurtubise-HMH, 1981.

Hendrykowska, Malgorzata. "Professeur, acteur ou bouffon? Le bonimenteur sur les territoires polonais avant 1914». Iris, n" 22 (1996), p. 161-181.

Komatsu, Hiroshi, et F. Loden. «Maîtriser l'image muette: le rôle des benshis dans le cinéma japonais». Iris, n" 22 (1996).

Koszarski, Richard. History of the American Cinema, vol. 3. An Evening's Entertainment: The Age of the Silent Feature Picture, 1915-1928. New York: Charles Scribner's Sons, 1990.

Leyda, Jay. Kino. Histoire du cinéma russe et soviétique. Lausanne: L'Âge d'homme, 1976.

Lyotard, Jean-François. Le Différend. Paris: Minuit, 1981. 
McLuhan, Marshall. Pour comprendre les médias. Les prolongements technologiques de L'homme. Montréal: Hurtubise-HMH, 1972.

Musser, Charles. "Portrait de l'exploitant des temps hérö̈ques en créateur». Film Échange, n" 25 (1984), p. 9-20.

Odin, Roger. "Pour une sémio-pragmatique du cinéma». Iris, vol. 1, n" 1 (1983), p. 67-83.

Pétrie, Juliette. Quand on revoit tout ça! Le burlesque au Québec. Montréal: Éditions Juliette Pétrie, 1977.

Prieur, Jérôme. Le Spectateur nocturne. Les écrivains au cinéma. Une anthologie. Paris: Cahiers du cinéma, 1993.

Saïdi, Mpungu Mulenda, Un regard en marge. Le public populaire du cinéma au Zaüre (thèse de doctorat). Louvain: Université catholique de Louvain, 1987. 